PROCEEDINGS OF THE

AMERICAN MATHEMATICAL SOCIETY

Volume 138, Number 8, August 2010, Pages 2961-2971

S 0002-9939(10)10330-X

Article electronically published on March 17, 2010

\title{
UNIFORMIZING LADDER SYSTEM COLORINGS AND THE RECTANGLE REFINING PROPERTY
}

\author{
TERUYUKI YORIOKA
}

(Communicated by Julia Knight)

\begin{abstract}
We investigate forcing notions with the rectangle refining property, which is stronger than the countable chain condition, and fragments of Martin's Axiom for such forcing notions. We prove that it is consistent that every forcing notion with the rectangle refining property has precaliber $\aleph_{1}$ but $\mathrm{MA}_{\aleph_{1}}$ for forcing notions with the rectangle refining property fails.
\end{abstract}

\section{INTRODUCTION}

In the 1980s, Stevo Todorčević introduced and investigated some types of fragments of Martin's Axiom for $\aleph_{1}$ dense sets, denoted by $\mathrm{MA}_{\aleph_{1}}$, for example in 9 . In [11, Todorčević and Veličković proved the following theorem [11, Theorem 3.4]: $M A_{\aleph_{1}}$ is equivalent to the statement that all countable chain condition (ccc) forcing notions have precaliber $\aleph_{1}$; that is, for all ccc forcing notions $\mathbb{P}$, every uncountable subset of $\mathbb{P}$ has an uncountable subset which has the finite compatibility property (i.e. every finitely many member has a common extension).

In [15, the author introduced a new chain condition, called the property $R_{1, \aleph_{1}}$, which is stronger than the countable chain condition. A typical forcing notion with this property is $a(T)$, which is the forcing notion adding an antichain through an Aronszajn tree $T$ by finite approximations, and we note that $M A_{\aleph_{1}}$ restricted to forcing notions with the property $R_{1, \aleph_{1}}$ implies that every Aronszajn tree is special. The author proved that it is consistent that every forcing notion with the property $\mathrm{R}_{1, \aleph_{1}}$ has precaliber $\aleph_{1}$ and there exists a non-special Aronszajn tree; hence $\mathrm{MA}_{\aleph_{1}}$ restricted to forcing notions with the property $R_{1, \aleph_{1}}$ fails [15, Theorem 6.2]. This is quite different from Todorčević and Veličković's theorem [11, Theorem 3.4].

The aim of this paper is to show a similar result for the rectangle refining property; that is, it is consistent that every forcing notion with the rectangle refining property has precaliber $\aleph_{1}$ and $\mathrm{MA}_{\aleph_{1}}$ restricted to forcing notions with the rectangle refining property fails. The rectangle refining property is introduced by Larson and Todorčević in [7] for partitions on $\left[\omega_{1}\right]^{2}$, and they introduced the axiom $\mathcal{K}_{2}$ (rec) which is the statement that every partition with the rectangle refining property has

Received by the editors July 27, 2009 and, in revised form, December 6, 2009.

2010 Mathematics Subject Classification. Primary 03E50, 03E05, $03 E 35$.

Key words and phrases. A uniformization of ladder system colorings, the rectangle refining property, fragments of Martin's Axiom.

The author was supported by Grant-in-Aid for Young Scientists (B), No. 19740048, Ministry of Education, Culture, Sports, Science and Technology.

(C)2010 American Mathematical Society 
an uncountable homogeneous set. In [12, the author introduced a property for forcing notions (called the anti-rectangle refining property) which is closely related to the rectangle refining property for partitions, and in [15], he introduced the rectangle refining property for forcing notions. In [15], there are some examples. A typical forcing notion with this property is also $a(T)$. We note that if $T$ is a Suslin tree, then $a(T)$ does not have property $\mathrm{K}$; in particular, $a(T)$ does not have precaliber $\aleph_{1}$. In [13], the author introduced a forcing notion with the rectangle refining property which is related to an unbounded family of the structure $\left(\omega^{\omega},<^{*}\right)$, and using this forcing notion, it was proved that $\mathcal{K}_{2}(\mathrm{rec})$ implies that the bounding number is larger than $\aleph_{1}$. A remarkable point about the rectangle refining property (and also the property $R_{1, \aleph_{1}}$ ) is that forcing notions with the rectangle refining property (or the property $R_{1, \aleph_{1}}$ ) add no random reals [15, §5]. It is unknown whether the rectangle refining property and the property $R_{1, \aleph_{1}}$ are different or not.

To show the main result, we investigate the following two concepts: uniformizations of ladder system colorings and forcing with a coherent Suslin tree.

In [1, Devlin and Shelah introduced a weak version of $\diamond$ and its applications to Whitehead's problem and uniformizations of ladder system colorings. (In the present paper, ladder systems means those on $\omega_{1}$. For details of ladder systems, see 93 .) The uniformization property of ladder system colorings plays a crucial role in Whitehead's problem. In fact, Eklof and Shelah proved in 2 that there exists a ladder system all of whose 2-colorings can be uniformized iff there exists a nonfree Whitehead group of size $\aleph_{1}$. In [1], Devlin and Shelah proved the following theorem [1, Theorem 5.2]: $\mathrm{MA}_{\aleph_{1}}$ implies that every ladder system coloring can be uniformized. We note that their forcing notions to uniformize a ladder system coloring has precaliber $\aleph_{1}$. In 93 , we give a forcing notion with the rectangle refining property which uniformizes a given ladder system coloring. In [12, §4] and [15, §4], it was proved that $\mathrm{MA}_{\aleph_{1}}$ restricted to forcing notions with the rectangle refining property is strictly weaker than $\mathrm{MA}_{\aleph_{1}}$. So the result in 93 refines Devlin and Shelah's theorem [1, Theorem 5.2].

In 10, Todorčević introduced the model $\operatorname{PFA}(S)[S] . \operatorname{PFA}(S)$ is an axiom which asserts that there exists a coherent Suslin tree $S$ such that the forcing axiom holds for every proper forcing notion which preserves $S$ as a Suslin tree; that is, if $\mathbb{P}$ is a proper forcing notion which preserves $S$ as a Suslin tree and $\left\{D_{\alpha} ; \alpha \in \omega_{1}\right\}$ is a set of $\aleph_{1}$-many dense open subsets of $\mathbb{P}$, then there exists a filter of $\mathbb{P}$ which intersects all of the $D_{\alpha}$ 's. PFA $(S)[S]$ is a forcing extension with this $S$ under $\operatorname{PFA}(S)$. We note that if there exists a supercompact cardinal, $\operatorname{PFA}(S)$ can be forced by the use of the property which is introduced by Miyamoto in $[8]$. PFA $(S)[S]$ is used to solve Katětov's problem, which is a question of set theoretic topology, in [5], and the model has some interesting properties. For example, in PFA $(S)[S]$, OCA holds $([3]), \mathcal{K}_{2}$ (rec) holds ([7]), and every Aronszajn tree is special. In particular, we should note that all of the above examples are satisfied in $\operatorname{MA}_{\aleph_{1}}(S)[S]$, which is made the same as $\operatorname{PFA}(S)[S]$ by replacing the properness with the countable chain condition. In [6, Theorem 6.2.], Larson and Todorčević proved that in the extension with a Suslin tree, every ladder system has a coloring which cannot be uniformized.

In 84 it is proved that it is consistent that there exists a coherent Suslin tree which forces the statement that every forcing notion with the rectangle refining property has precaliber $\aleph_{1}$ (Theorem 4.1). This is witnessed by the model of the form $\mathrm{MA}_{\aleph_{1}}(S)[S]$ as well as of the form $\operatorname{PFA}(S)[S]$. Therefore by the result in 
$\S 3$ and Larson and Todorčević's theorem [6, Theorem 6.2.], $\mathrm{MA}_{\aleph_{1}}$ restricted to forcing notions with the rectangle refining property fails in $\operatorname{MA}_{\aleph_{1}}(S)[S]$ and also in $\operatorname{PFA}(S)[S]$.

The conclusion of Theorem 4.1 is different from [15, Theorem 6.2.]. Because in the case [15, Theorem 6.2.], it is necessary that there exists a non-special Aronszajn tree in the extension to guarantee that $\mathrm{MA}_{\aleph_{1}}$ for forcing notions with the property $\mathrm{R}_{1, \aleph_{1}}$ fails; however, in the case Theorem 4.1, by considering $\mathrm{MA}_{\aleph_{1}}(S)[S]$, it is possible that all Aronszajn trees are special in the extension.

\section{The Class $\mathrm{FSCO}_{s}$ AND THE RECTANGLE REFINING PROPERTY}

In this paper, as in [15], forcing notions mean partial orders, and we focus on forcing notions of size $\aleph_{1}$ because we will study fragments of $\mathrm{MA}_{\aleph_{1}}$, Martin's Axiom for $\aleph_{1}$ many dense sets. We consider the following types of forcing notions, which are slightly different from FSCO in [15] (see below).

Definition 2.1. $\mathrm{FSCO}_{0}$ is the collection of forcing notions $\mathbb{P}$ such that

- a condition of $\mathbb{P}$ is a finite set of countable ordinals,

- $\mathbb{P}$ is uncountable, and

- $\leq_{\mathbb{P}}$ is equal to the superset relation $\supseteq$; that is, for any $\sigma$ and $\tau$ in $\mathbb{P}, \sigma \leq_{\mathbb{P}} \tau$ iff $\sigma \supseteq \tau$.

Many applications of $\mathrm{MA}_{\aleph_{1}}$ use these types of forcing notions; see [15. FSCO in [15] is the set of forcing notions in $\mathrm{FSCO}_{0}$ with the additional property that $\mathbb{P}$ is closed under subsets; that is, if $\sigma \in \mathbb{P}$ and $\tau \subseteq \sigma$, then $\tau \in \mathbb{P}$. So $\mathrm{FSCO}_{0}$ is a wider class than FSCO. In this paper, we deal with the subclass of $\mathrm{FSCO}_{0}$ as follows.

Definition 2.2. $\mathrm{FSCO}_{1}$ is the collection of forcing notions $\mathbb{P}$ in $\mathrm{FSCO}_{0}$ such that for any uncountable subset $I$ of $\mathbb{P}$, there exists an uncountable subset $I^{\prime}$ of $I$ such that for every finite subset $\rho$ of $I^{\prime}$, if $\rho$ has a common extension in $\mathbb{P}, \bigcup \rho$ is one of its common extensions.

$\mathrm{FSCO}_{1}$ is a wider class than FSCO. If $\mathbb{P}$ is in $\mathrm{FSCO}$, since $\mathbb{P}$ is closed under subsets and if a finite subset $\rho$ of $\mathbb{P}$ has a common extension, then $\bigcup \rho$ is its common extension. So $\mathbb{P}$ belongs to $\mathrm{FSCO}_{1}$.

Definition 2.3. $\mathrm{FSCO}_{2}$ is the collection of forcing notions $\mathbb{P}$ in $\mathrm{FSCO}_{1}$ such that for any uncountable subset $\left\{\sigma_{\alpha} ; \alpha \in \omega_{1}\right\}$ of $\mathbb{P}$, there are an uncountable subset $\Gamma$ of $\omega_{1}$ and a sequence $\left\langle\sigma_{\alpha}^{\prime} ; \alpha \in \Gamma\right\rangle$ such that

- for each $\alpha \in \Gamma, \sigma_{\alpha}^{\prime} \leq_{\mathbb{P}} \sigma_{\alpha}$ (i.e. $\sigma_{\alpha}^{\prime} \supseteq \sigma_{\alpha}$ ),

- the set $\left\{\sigma_{\alpha}^{\prime} ; \alpha \in \omega_{1}\right\}$ forms a $\Delta$-system, and

- for every finite subset $\rho$ of $\Gamma$, if the set $\left\{\sigma_{\alpha}^{\prime} ; \alpha \in \rho\right\}$ has a common extension in $\mathbb{P}$, then $\bigcup_{\alpha \in \rho} \sigma_{\alpha}^{\prime}$ is its common extension and the set

$$
\left\{\beta \in \Gamma ;\left\{\sigma_{\alpha}^{\prime} ; \alpha \in \rho\right\} \cup\left\{\sigma_{\beta}^{\prime}\right\} \text { has a common extension in } \mathbb{P}\right\}
$$

is uncountable.

The definition of $\mathrm{FSCO}_{2}$ seems to be technical. But it follows from the next claim that $\mathrm{FSCO}_{2}$ is also wider than the class of ccc forcing notions in FSCO.

Claim 2.4. If $\mathbb{P}$ is a forcing notion in FSCO with the countable chain condition, then $\mathbb{P}$ belongs to $\mathrm{FSCO}_{2}$. 
Proof. Here, we note again that if a finite subset $\rho$ of $\mathbb{P}$ has a common extension in $\mathbb{P}$, then $\bigcup \rho$ is its common extension in $\mathbb{P}$, because $\mathbb{P}$ is closed under subsets.

Let $\left\{\sigma_{\alpha} ; \alpha \in \omega_{1}\right\}$ be an uncountable subset of $\mathbb{P}$. We take an uncountable subset $\Gamma$ of $\omega_{1}$ such that the set $\left\{\sigma_{\alpha} ; \alpha \in \Gamma\right\}$ forms a $\Delta$-system. Since $\mathbb{P}$ is ccc, there exists $\tau \in \mathbb{P}$ such that

$$
\tau \Vdash_{\mathbb{P}} "\left\{\sigma_{\alpha} ; \alpha \in \Gamma\right\} \cap \dot{G} \text { is uncountable ". }
$$

Then the set $\left\{\sigma_{\alpha} \cup \tau ; \alpha \in \Gamma\right\} \cap \mathbb{P}$ are as desired.

The following figure is a relationship between the subclasses of $\mathrm{FSCO}_{0}$.

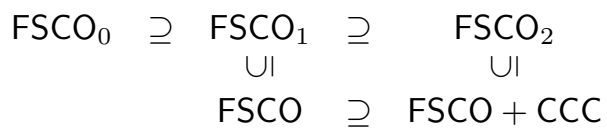

Definition 2.5. A forcing notion $\mathbb{P}$ in $\mathrm{FSCO}_{0}$ has the rectangle refining property if for any pair of uncountable subsets $I$ and $J$ of $\mathbb{P}$, whenever $I \cup J$ forms a $\Delta$-system, then there are uncountable subsets $I^{\prime}$ and $J^{\prime}$ of $I$ and $J$ respectively such that any member of $I^{\prime}$ is compatible with any member of $J^{\prime}$ in $\mathbb{P}$.

A typical forcing notion with this property is also $a(T)$. In [14, the author introduced the rectangle refining property for general partial orders. It is easy to see that a specializing Aronszajn tree by finite approximations has the rectangle refining property in the sense of [14]. The definition in 14 includes that of the present paper (for other examples, see [12, 13, 15]).

\section{UNIFORMIZING A LADDER SYSTEM COLORING}

A ladder system on $\omega_{1}$ is a sequence $\left\langle C_{\alpha} ; \alpha \in \omega_{1} \cap \operatorname{Lim}\right\rangle$ such that for each $\alpha \in$ $\omega_{1} \cap \operatorname{Lim}, C_{\alpha}$ is an unbounded subset of $\alpha$ and the order type of $C_{\alpha}$ is $\omega$. A coloring of the ladder system $\left\langle C_{\alpha} ; \alpha \in \omega_{1} \cap \operatorname{Lim}\right\rangle$ is a sequence $\left\langle f_{\alpha} ; \alpha \in \omega_{1} \cap \operatorname{Lim}\right\rangle$ such that for each $\alpha \in \omega_{1} \cap \operatorname{Lim}, f_{\alpha}$ is a function from $C_{\alpha}$ into $\omega$. We say that a function $\varphi$ from $\omega_{1}$ into $\omega$ uniformizes the coloring $\left\langle f_{\alpha} ; \alpha \in \omega_{1} \cap \operatorname{Lim}\right\rangle$ if for every $\alpha \in \omega_{1} \cap \operatorname{Lim}$, the difference between $f_{\alpha}$ and $\varphi \uparrow \operatorname{dom}\left(f_{\alpha}\right)$ is finite.

Let $\left\langle C_{\alpha} ; \alpha \in \omega_{1} \cap \operatorname{Lim}\right\rangle$ be a ladder system, let $\left\langle\eta_{n}^{\alpha} ; n \in \omega\right\rangle$ be an increasing enumeration of $C_{\alpha}$ for each $\alpha \in \omega_{1} \cap \operatorname{Lim}$, and let $\left\langle f_{\alpha} ; \alpha \in \omega_{1} \cap \operatorname{Lim}\right\rangle$ be a coloring of this ladder. Then let

$$
\mathbb{P}\left(\left\langle C_{\alpha} ; \alpha \in \omega_{1} \cap \operatorname{Lim}\right\rangle,\left\langle f_{\alpha} ; \alpha \in \omega_{1} \cap \operatorname{Lim}\right\rangle\right)
$$

be a forcing notion which consists of finite partial functions $p$ from $\omega_{1} \cap$ Lim into $\omega$ such that the set

$$
\bigcup_{\gamma \in \operatorname{dom}(p)}\left(f_{\gamma}\left\lceil\left\{\eta_{n}^{\gamma} ; n \geq p(\gamma)\right\}\right)\right.
$$

forms a function, and this is ordered by the reverse inclusion (in this section, it is denoted by $\mathbb{P}$ for short). We note that $\mathbb{P}$ can be considered as a forcing notion in FSCO, $\mathbb{P}$ adds a uniformizing function for this coloring, $\mathbb{P}$ has precaliber $\aleph_{1}$, but in general, $\mathbb{P}$ doesn't have the rectangle refining property. (For example, if there exist $n \in \omega$ and disjoint uncountable subsets $I$ and $J$ of $\omega_{1} \cap \operatorname{Lim}$ such that the set $\left\{\eta_{n}^{\alpha} ; \alpha \in I \cup J\right\}$ is a singleton and for every $\alpha \in I$ and $\beta \in J, f_{\alpha}\left(\eta_{n}^{\alpha}\right) \neq f_{\beta}\left(\eta_{n}^{\beta}\right)$, then the pair of sets $\{\{\langle\alpha, n\rangle\} ; \alpha \in I\}$ and $\{\{\langle\beta, n\rangle\} ; \beta \in J\}$ is a witness which guarantees that $\mathbb{P}$ doesn't have the rectangle refining property.) 
We define another forcing notion for uniformizing a coloring. For a subset $\sigma$ of the set $\{0,1\} \times X$ and $i \in\{0,1\}$, let

$$
\pi_{i}[\sigma]:=\{x \in X ;\langle i, x\rangle \in \sigma\} .
$$

The forcing notion

$$
\mathbb{P}^{2}\left(\left\langle C_{\alpha} ; \alpha \in \omega_{1} \cap \operatorname{Lim}\right\rangle,\left\langle f_{\alpha} ; \alpha \in \omega_{1} \cap \operatorname{Lim}\right\rangle\right)
$$

consists of subsets $\sigma$ of the set

$$
\left(\{0\} \times\left(\left(\omega_{1} \cap \operatorname{Lim}\right) \times \omega\right)\right) \cup\left(\{1\} \times\left(\omega_{1} \times \omega\right)\right)
$$

such that

- $\pi_{0}[\sigma] \in \mathbb{P}\left(\left\langle C_{\alpha} ; \alpha \in \omega_{1} \cap \operatorname{Lim}\right\rangle,\left\langle f_{\alpha} ; \alpha \in \omega_{1} \cap \operatorname{Lim}\right\rangle\right)$,

- $\pi_{1}[\sigma]$ is the set

$$
\begin{aligned}
& \left\{\left\langle\eta_{\pi_{0}[\sigma](\gamma)}^{\gamma}, f_{\gamma}\left(\eta_{\pi_{0}[\sigma](\gamma)}^{\gamma}\right)\right\rangle ; \gamma \in \operatorname{dom}\left(\pi_{0}[\sigma]\right)\right\} \\
& \cup\left\{\left\langle\eta_{n}^{\gamma}, f_{\gamma}\left(\eta_{n}^{\gamma}\right)\right\rangle ; \gamma \in \operatorname{dom}\left(\pi_{0}[\sigma]\right) \text { and } n \in \omega \backslash \pi_{0}[\sigma](\gamma)\right. \\
& \left.\quad \text { such that } \exists \gamma^{\prime} \in \operatorname{dom}\left(\pi_{0}[\sigma]\right) \cap \gamma\left(\eta_{n}^{\gamma}<\gamma^{\prime}\right)\right\}
\end{aligned}
$$

(so $\pi_{1}[\sigma]$ is also a finite function),

and this is ordered by the reverse inclusion (in this section, it is denoted by $\mathbb{P}^{2}$ for short).

$\mathbb{P}^{2}$ can also be considered as a forcing notion in FSCO and almost the same as $\mathbb{P}$. The difference is that a condition of $\mathbb{P}^{2}$ has information of finite pieces of the coloring which is necessary to think about the compatibility of the conditions of $\mathbb{P}$ (and also $\mathbb{P}^{2}$ ).

Lemma 3.1. $\mathbb{P}^{2}$ is a forcing notion in $\mathrm{FSCO}_{2}$ with the rectangle refining property.

Proof. By the definition, $\mathbb{P}^{2}$ is a forcing notion in $\mathrm{FSCO}_{0}$. At first, we show that $\mathbb{P}^{2}$ has the rectangle refining property. The idea of this proof says that $\mathbb{P}^{2}$ belongs to $\mathrm{FSCO}_{1}$.

Let $I$ and $J$ be disjoint uncountable subsets of $\omega_{1}$, and let $\left\{\sigma_{\alpha} ; \alpha \in I \cup J\right\}$ be an uncountable subset of $\mathbb{P}^{2}$ which forms a $\Delta$-system. Let $R$ be the root of this $\Delta$-system. Let $\delta$ be a countable ordinal such that $R \subseteq\{0,1\} \times(\delta \times \omega)$. Then we can find uncountable subsets $I^{\prime}$ and $J^{\prime}$ of $I$ and $J$ respectively such that

(a) $\left\{\sigma_{\alpha} ; \alpha \in I^{\prime}\right\} \cap\left\{\sigma_{\alpha} ; \alpha \in J^{\prime}\right\}=\emptyset$,

(b) for any $\alpha$ and $\beta$ in the set $I^{\prime} \cup J^{\prime}$, if $\alpha<\beta$, then

$$
\delta<\min \left(\left(\operatorname{dom}\left(\pi_{0}\left[\sigma_{\alpha}\right]\right) \backslash \delta\right) \cup\left(\operatorname{dom}\left(\pi_{1}\left[\sigma_{\alpha}\right]\right) \backslash \delta\right)\right)
$$

and

$$
\max \left(\pi_{0}\left[\sigma_{\alpha}\right]\right)<\min \left(\left(\operatorname{dom}\left(\pi_{0}\left[\sigma_{\beta}\right]\right) \backslash \delta\right) \cup\left(\operatorname{dom}\left(\pi_{1}\left[\sigma_{\beta}\right]\right) \backslash \delta\right)\right) .
$$

Then we notice that the set $\left\{\sigma_{\alpha} ; \alpha \in I^{\prime} \cup J^{\prime}\right\}$ still forms a $\Delta$-system with the root $R$, and the function

$$
\bigcup_{\gamma \in \operatorname{dom}\left(\sigma_{\alpha}\right)}\left(f_{\gamma} \uparrow\left(\left\{\eta_{n}^{\gamma} ; n \geq \pi_{0}\left[\sigma_{\alpha}\right](\gamma)\right\} \cap \delta\right)\right)
$$


doesn't depend on $\alpha \in I^{\prime} \cup J^{\prime}$. Moreover since the domain of the function

$$
\bigcup_{\gamma \in \operatorname{dom}\left(\sigma_{\alpha}\right)}\left(f_{\gamma}\left\lceil\left\{\eta_{n}^{\gamma} ; n \geq \pi_{0}[\sigma](\gamma)\right\}\right)\right.
$$

is a subset of the set

$$
\delta \cup\left[\min \left(\left(\operatorname{dom}\left(\pi_{0}\left[\sigma_{\alpha}\right]\right) \backslash \delta\right) \cup\left(\operatorname{dom}\left(\pi_{1}\left[\sigma_{\alpha}\right] \backslash \delta\right)\right)\right), \max \left(\operatorname{dom}\left(\pi_{0}\left[\sigma_{\alpha}\right]\right)\right)\right)
$$

for every $\alpha \in I^{\prime} \cup J^{\prime}$ (here, for two ordinals $\xi$ and $\eta,[\xi, \eta$ ) is the interval of ordinals; that is, $[\xi, \eta):=\{\zeta \in \eta ; \xi \leq \zeta\}), \sigma_{\alpha}$ and $\sigma_{\beta}$ are compatible in $\mathbb{P}^{2}$ for any $\alpha \in I^{\prime}$ and $\beta \in J^{\prime}$. In fact, for any finite subset $\rho$ of $I^{\prime} \cup J^{\prime}, \bigcup_{\alpha \in \rho} \sigma_{\alpha}$ is a common extension of the $\sigma_{\alpha}$ 's in $\mathbb{P}^{2}$. (This argument also says that $\mathbb{P}^{2}$ also has precaliber $\aleph_{1}$.)

To show that $\mathbb{P}^{2}$ belongs to $\mathrm{FSCO}_{2}$, let $\left\{\sigma_{\alpha} ; \alpha \in \omega_{1}\right\}$ be an uncountable subset of $\mathbb{P}^{2}$. We may assume that there exists $\delta \in \omega_{1}$ such that $\left\{\sigma_{\alpha} ; \alpha \in \omega_{1}\right\}$ forms a $\Delta$ system with a root which is a subset of the set $\{0,1\} \times \delta$, and $\left\{\sigma_{\alpha} ; \alpha \in \omega_{1}\right\}$ satisfies the property (b) above.

Since $\mathbb{P}^{2}$ has the rectangle refining property (in particular the countable chain condition), there exists $\tau \in \mathbb{P}^{2}$ such that

$$
\tau \Vdash_{\mathbb{P}^{2}} \text { " }\left\{\sigma_{\alpha} ; \alpha \in \omega_{1}\right\} \cap \dot{G} \text { is uncountable ". }
$$

Take $\alpha \in \omega_{1}$ such that $\sigma_{\alpha}$ and $\tau$ are compatible in $\mathbb{P}^{2}$, and let $\tau^{\prime}$ be an extension of $\tau$ in $\mathbb{P}^{2}$ such that $\pi_{0}\left[\tau^{\prime}\right]=\pi_{0}[\tau]$ and $\sigma_{\alpha} \cup \tau^{\prime}$ is a condition of $\mathbb{P}^{2}$ (hence it is a common extension of $\sigma_{\alpha}$ and $\tau$ ). Then we note that for each $\beta \in \omega_{1}$ larger than $\alpha, \sigma_{\beta}$ and $\tau$ are compatible in $\mathbb{P}^{2}$ iff $\sigma_{\beta} \cup \tau^{\prime}$ is a condition in $\mathbb{P}^{2}$. Therefore the set $\left\{\sigma_{\beta} \cup \tau^{\prime} ; \beta \in \omega_{1} \backslash \alpha\right\} \cap \mathbb{P}^{2}$ is as desired.

\section{Forcing With a coherent Suslin tree}

This section contains a proof of the following theorem.

Theorem 4.1. It is consistent that there exists a coherent Suslin tree which forces the statement that every forcing notion in $\mathrm{FSCO}_{2}$ with thathe rectangle refining property has precaliber $\aleph_{1}$.

Throughout this section, we suppose that $S$ is a coherent Suslin tree which consists of functions in $\omega^{<\omega_{1}}$ and is closed under finite modifications. That is,

- for any $s$ and $t$ in $S, s \leq_{S} t$ iff $s \subseteq t$,

- $S$ is closed under taking initial segments,

- for any $s$ and $t$ in $S$, the set

$$
\{\alpha \in \min \{\operatorname{lv}(s), \operatorname{lv}(t)\} ; s(\alpha) \neq t(\alpha)\}
$$

is finite (here, $\operatorname{lv}(s)$ is the length of $s$, that is, the size of $s$, which represents the level of $s$ in the tree $S$ ), and

- for any $s \in S$ and $t \in \omega^{\operatorname{lv}(s)}$, if the set $\{\alpha \in \operatorname{lv}(s) ; s(\alpha) \neq t(\alpha)\}$ is finite, then $t \in S$ also.

For a countable ordinal $\alpha$, let $S_{\alpha}$ be the set of $\alpha$-th level nodes, that is, the set of all members of $S$ of domain $\alpha$, and let $S_{\leq \alpha}:=\bigcup_{\beta \leq \alpha} S_{\beta}$. For $s \in S$, we let

$$
S\left\lceil s:=\left\{u \in S ; s \leq_{S} u\right\} .\right.
$$

We note that $\diamond$, or adding a Cohen real, builds a coherent Suslin tree. A coherent Suslin tree has canonical commutative isomorphisms. Let $s$ and $t$ be nodes in $S$ 
with the same level. Then we define a function $\psi_{s, t}$ from $S\lceil s$ into $S\lceil t$ such that for each $v \in S\lceil s$,

$$
\psi_{s, t}(v):=t \cup(v\lceil[\operatorname{lv}(s), \operatorname{lv}(v)))
$$

(here, $v\lceil[\operatorname{lv}(s), \operatorname{lv}(v))$ is the function $v$ restricted to the domain $[\operatorname{lv}(s), \operatorname{lv}(v))$ ). We note that $\psi_{s, t}$ is an isomorphism, and if $s, t, u$ are nodes in $S$ with the same level, then $\psi_{s, t}, \psi_{t, u}$ and $\psi_{s, u}$ commute. (On a coherent Suslin tree, see e.g. [4, 6.)

Let $\dot{\mathbb{P}}$ be an $S$-name for a forcing notion in $\mathrm{FSCO}_{2}$ with the rectangle refining property, $\dot{I}=\left\{\dot{\sigma}_{\alpha} ; \alpha \in \omega_{1}\right\} S$-names for conditions of $\dot{\mathbb{P}}$ which forms a $\Delta$-system such that

$$
\begin{gathered}
\Vdash_{T} \text { " for any } \rho \in\left[\omega_{1}\right]^{<\aleph_{0}} \text {, if the set }\left\{\dot{\sigma}_{\alpha} ; \alpha \in \rho\right\} \text { has a common extension in } \dot{\mathbb{P}}, \\
\text { then } \bigcup_{\alpha \in \rho} \dot{\sigma}_{\alpha} \in \mathbb{P} \text { and } \\
\left\{\beta \in \omega_{1} ;\left\{\dot{\sigma}_{\alpha} ; \alpha \in \rho\right\} \cup\left\{\dot{\sigma}_{\beta}\right\} \text { has a common extension }\right\} \text { is uncountable". }
\end{gathered}
$$

Since $S$ has the countable chain condition, we can find a club $C$ on $\omega_{1}$ such that for every $\alpha \in C$, every node in $S_{\alpha}$ decides all of the names $\dot{\sigma}_{\beta}$, for every $\beta<\alpha$, $\dot{\mathbb{P}} \cap[\alpha]^{<\aleph_{0}}$ and $\leq_{\dot{\mathbb{P}}} \cap\left([\alpha]^{<\aleph_{0}} \times[\alpha]^{<\aleph_{0}}\right)$.

Let $\mathbb{Q}_{S}(\dot{\mathbb{P}}, \dot{I}, C)$ be the forcing notion which consists of finite partial functions $f$ from $S$ into $\left[\omega_{1}\right]^{<\aleph_{0}}$ such that for each $t \in \operatorname{dom}(f), f(t) \subseteq C \cap \operatorname{lv}(t)$, and

$$
t \Vdash \text { " }\left\{\dot{\sigma}_{\gamma} ; \exists t^{\prime} \in \operatorname{dom}(f)\left(t^{\prime} \leq_{S} t \& \gamma \in f\left(t^{\prime}\right)\right)\right\} \in \dot{\mathbb{P}} "
$$

(which is equivalent to

$$
t \Vdash \text { “ }\left\{\dot{\sigma}_{\gamma} ; \exists t^{\prime} \in \operatorname{dom}\left(f_{\xi}\right)\left(t^{\prime} \leq_{S} t \& \gamma \in f\left(t^{\prime}\right)\right)\right\} \text { has a common extension "), }
$$

and this is ordered by the reverse inclusion.

Suppose that $\mathbb{Q}_{S}(\dot{\mathbb{P}}, \dot{I}, C)$ has the countable chain condition and it preserves $S$ to be a Suslin tree. Then we note that, by the property of $\dot{I}$, if $G$ is $\mathbb{Q}_{S}(\dot{\mathbb{P}}, \dot{I}, C)$-generic, then $\bigcup G$ is a partial function such that for every $t \in \operatorname{dom}(\bigcup G)$ and $\alpha \in \omega_{1}$, there exists $t^{\prime} \in \operatorname{dom}(\bigcup G)$ such that $t \leq_{S} t^{\prime}$ and $(\bigcup G)\left(t^{\prime}\right) \nsubseteq \subset \alpha$. Therefore in the extension with $\mathbb{Q}_{S}(\dot{\mathbb{P}}, \dot{I}, C), S$ generically adds an uncountable set $J$ of countable ordinals such that, in the extension by an $S$-generic filter $G_{S}$, the set $\left\{\dot{\sigma}_{\alpha}\left[G_{S}\right] ; \alpha \in J\right\}$ has the finite compatibility property. Therefore by considering the book-keeping argument of a finite support iteration or considering $\mathrm{MA}_{\aleph_{1}}(S)[S]$, we only have to show that $\mathbb{Q}_{S}(\dot{\mathbb{P}}, \dot{I}, C)$ has the countable chain condition and preserves $S$ to be Suslin. It suffices to prove that $\mathbb{Q}_{S}(\dot{\mathbb{P}}, \dot{I}, C) \times S$ has the countable chain condition. In [7, Theorem 4.2], Larson and Todorčević proved that a coherent Suslin tree may force $\mathcal{K}_{2}(\mathrm{rec})$. The idea of the following proof is similar to their proof. The author used a similar technique in [14, §3]. But the proof below is much simpler than ones in [14, §3], because here we deal with much simpler structures than those in [14, §3].

Lemma 4.2. For any $S, \dot{\mathbb{P}}, \dot{I}$ and $C$ as above, $\mathbb{Q}_{S}(\dot{\mathbb{P}}, \dot{I}, C) \times S$ has the countable chain condition.

Proof. In this proof, $\mathbb{Q}_{S}(\dot{\mathbb{P}}, \dot{I}, C)$ is denoted by $\mathbb{Q}_{S}$ for short. Let $\left\{\left\langle f_{\xi}, t_{\xi}\right\rangle ; \xi \in \omega_{1}\right\}$ be an uncountable subset of $\mathbb{Q}_{S} \times S$. By strengthening conditions if necessary, we may assume that for every $\xi \in \omega_{1}$,

$$
t_{\xi} \in \operatorname{dom}\left(f_{\xi}\right) \cap S_{\max \left(\operatorname{lv}\left[\operatorname{dom}\left(f_{\xi}\right)\right]\right) .} .
$$


By strengthening conditions again and shrinking the set $\left\{\left\langle f_{\xi}, t_{\xi}\right\rangle ; \xi \in \omega_{1}\right\}$ if necessary, we may moreover assume that there are $\delta \in \omega_{1}, m, n \in \omega(m \leq n)$ and $s \in S$ such that

- the set $\left\{\operatorname{dom}\left(f_{\xi}\right) ; \xi \in \omega_{1}\right\}$ forms a $\Delta$-system with root which is a subset of $S_{\leq \delta}$

- for every $\xi$ and $\eta$ in $\omega_{1}$,

$$
\operatorname{dom}\left(f_{\xi}\right) \cap S_{\leq \delta}=\operatorname{dom}\left(f_{\eta}\right) \cap S_{\leq \delta} \text { and } f_{\xi}\left\lceil S_{\leq \delta}=f_{\eta}\left\lceil S_{\leq \delta}\right.\right.
$$

- for each $\xi \in \omega_{1}$, letting $\alpha_{\xi}:=\max \left(\operatorname{Iv}\left[\operatorname{dom}\left(f_{\xi}\right)\right]\right)$, every node of the set $\operatorname{dom}\left(f_{\xi}\right) \backslash S_{\alpha_{\xi}}$ (which is equal to $\operatorname{dom}\left(f_{\xi}\right) \cap S_{<\alpha_{\xi}}$ ) is below some node of the set $\operatorname{dom}\left(f_{\xi}\right) \cap S_{\alpha_{\xi}}$;

- for each $\xi$ in $\omega_{1}$, the size of the set $\operatorname{dom}\left(f_{\xi}\right) \cap S_{\alpha_{\xi}}$ is $n$, and let

$$
\operatorname{dom}\left(f_{\xi}\right) \cap S_{\alpha_{\xi}}=\left\{t_{i}^{\xi} ; i \in n\right\}
$$

- there exists a sequence $\left\langle\alpha_{\xi}^{\prime} ; \xi \in \omega_{1}\right\rangle$ of countable ordinals such that for each $\xi$ and $\eta$ in $\omega_{1}$ with $\xi<\eta$,

$$
\delta<\alpha_{\xi}^{\prime} \leq \min \left(\operatorname{lv}\left[\operatorname{dom}\left(f_{\xi}\right) \backslash S_{\leq \delta}\right]\right)<\alpha_{\xi}=\max \left(\operatorname{lv}\left[\operatorname{dom}\left(f_{\xi}\right)\right]\right)<\alpha_{\eta}^{\prime}
$$

and

$$
\left\{\beta \in \omega_{1} ; \exists i, j \in m\left(t_{i}^{\xi}(\beta) \neq t_{j}^{\xi}(\beta)\right)\right\} \subseteq \delta \cup\left[\alpha_{\xi}^{\prime}, \alpha_{\xi}\right) ;
$$

- for each $\xi$ in $\omega_{1}, t_{\xi}=t_{0}^{\xi}$ and for every $j \in n$, there exists a unique $i \in m$ such that $t_{j}^{\xi}\left\lceil\alpha_{\xi}^{\prime}=t_{i}^{\xi}\left\lceil\alpha_{\xi}^{\prime}\right.\right.$ and $t_{i}^{\xi}\left\lceil\left[\alpha_{\xi}^{\prime}, \alpha_{\xi}\right)=t_{0}^{\xi} \uparrow\left[\alpha_{\xi}^{\prime}, \alpha_{\xi}\right)\right.$;

- for every $\xi$ and $\eta$ in $\omega_{1}$ and $i \in n, t_{i}^{\xi}\left\lceil\delta=t_{i}^{\eta}\lceil\delta\right.$;

- for each $i \in n$, the set

$$
\left\{\bigcup\left\{f_{\xi}(t) ; t \in \operatorname{dom}\left(f_{\xi}\right) \& t \leq_{S} t_{i}^{\xi}\right\} ; \xi \in \omega_{1}\right\}
$$

forms a $\Delta$-system with a root which is also a subset of $\delta$;

- $\operatorname{Iv}(s)=\delta$ holds, $s \leq_{S} t_{0}^{\xi}=t_{\xi}$ for every $\xi \in \omega_{1}$, and the set $\left\{t_{0}^{\xi} ; \xi \in \omega_{1}\right\}$ is dense above $s$.

For each $i \in n$ and $\xi \in \omega_{1}$, let

$$
s_{i}:=\psi_{s, t_{i}^{0} \operatorname{lv}(s)}(s)=t_{i}^{0} \operatorname{lv}(s)
$$

and

$$
\Vdash_{S} " \dot{\tau}_{i}^{\xi}:=\bigcup\left\{\dot{\sigma}_{\gamma} ; \exists t \in \operatorname{dom}\left(f_{\xi}\right)\left(t \leq_{S} t_{i}^{\xi} \& \gamma \in f_{\xi}(t)\right)\right\} " .
$$

Then by our $\Delta$-system refinement, for every $i \in n$ and $\xi \in \omega_{1}, s_{i}<_{S} t_{i}^{\xi}$ holds.

We enumerate the set $\left\{\langle i, j\rangle \in m \times n ; s_{i}=s_{j}\right\}$ by $\left\langle\left\langle i_{\mu}^{0}, i_{\mu}^{1}\right\rangle ; \mu \in N\right\rangle$ with

$$
\left\langle i_{N-1}^{0}, i_{N-1}^{1}\right\rangle=\langle 0,0\rangle,
$$

and we will recursively find $S$-names $\dot{K}_{\mu}^{0}$ and $\dot{K}_{\mu}^{1}$ and uncountable subsets $J_{\mu}^{0}$ and $J_{\mu}^{1}$ of $\omega_{1}$ such that $J_{-1}^{0}=J_{-1}^{1}=\omega_{1}$ and for each $\mu \in N$,

- for each $l \in\{0,1\}$ (note that $s_{i_{\mu}^{0}}=s_{i_{\mu}^{1}}$ ),

$$
s_{i_{\mu}^{0}} \vdash_{S} \text { " } \dot{K}_{\mu}^{l} \text { is an uncountable subset of the set }\left\{\dot{\tau}_{i_{\mu}^{l}}^{\xi} ; \xi \in J_{\mu-1}^{l}\right\} \text { "; }
$$


- for each $l \in\{0,1\}$ and any $\xi \in J_{\mu}^{l}$ and $u \in S$, if

$$
u \Vdash_{S} " \dot{\tau}_{i_{\mu}^{l}}^{\xi} \in \dot{K}_{\mu}^{l} ",
$$

then $t_{i_{\mu}^{l}}^{\xi} \leq_{S} u$ and for every $\mu^{\prime}<\mu$,

$$
\psi_{i_{i_{\mu}}^{\xi}, t_{i_{\mu^{\prime}}}^{\xi}}(u) \Vdash_{S} “ \dot{\tau}_{i_{\mu^{\prime}}^{l}}^{\xi} \in \dot{K}_{\mu^{\prime}}^{l} " ;
$$

- $s_{i_{\mu}^{0}} \Vdash_{S}{ }^{~ " ~} \dot{K}_{\mu}^{0} \cap \dot{K}_{\mu}^{1}=\emptyset$ and for every $\varepsilon \in \dot{K}_{\mu}^{0}$ and $\rho \in \dot{K}_{\mu}^{1}$, $\varepsilon$ and $\rho$ are compatible in $\dot{\mathbb{P}}$, i.e. $\varepsilon \cup \rho \in \dot{\mathbb{P}}$; and

- for each $l \in\{0,1\}$, let

$$
J_{\mu}^{l}:=\left\{\xi \in J_{\mu-1}^{l} ; \exists u \in T\left(u \Vdash_{S} " \dot{\tau}_{i_{\mu}^{l}}^{\xi} \in \dot{K}_{\mu}^{l} "\right)\right\} .
$$

We can construct them by induction on $\mu \in N$ as follows. At stage $\mu \in N$, we define $S$-names $\underset{\sim}{K_{\mu}^{0}}$ and $\underset{\sim}{K_{\mu}^{1}}$ such that for each $l \in\{0,1\}$,

$$
s_{i_{\mu}^{0}} \Vdash_{S} " \underset{\sim}{K_{\mu}^{l}} \subseteq\left\{\dot{\tau}_{i_{\mu}^{l}}^{\xi} ; \xi \in J_{\mu-1}^{l}\right\} ",
$$

and for any $\xi \in J_{\mu-1}^{l}$ and $u \in S$,

$$
u \Vdash_{S} " \dot{\tau}_{i_{\mu}^{l}}^{\xi} \in \underset{\sim}{K_{\mu}^{l}} ": \Longleftrightarrow t_{i_{\mu}^{l}}^{\xi} \leq_{S} u \& \forall \mu^{\prime}<\mu\left(\psi_{t_{i_{\mu}^{l}}^{\xi}, t_{i_{\mu^{\prime}}}^{\xi}}(u) \Vdash_{S} " \dot{\tau}_{i_{\mu^{\prime}}^{l}}^{\xi} \in \dot{K}_{\mu^{\prime}} "\right) .
$$

Since

$$
s_{i_{\mu}^{l}} \Vdash_{S} \text { “ } \underset{\sim}{K_{\mu}^{0}} \text { and } \underset{\sim}{K_{\mu}} \text { are uncountable and } \underset{\sim}{K_{\mu}^{0}} \cup \underset{\sim}{K_{\mu}^{1}} \text { forms a } \Delta \text {-system ", }
$$

there are $S$-names $\dot{K}_{\mu}^{0}$ and $\dot{K}_{\mu}^{1}$ for uncountable subsets of $\underset{\sim}{K_{\mu}^{0}}$ and $\underset{\sim}{K_{\mu}^{1}}$ respectively such that

$$
s_{i_{\mu}^{l}} \Vdash_{S} " \dot{K}_{\mu}^{0} \cap \dot{K}_{\mu}^{1}=\emptyset \text { and for every } \varepsilon \in \dot{K}_{\mu}^{0} \text { and } \rho \in \dot{K}_{\mu}^{1}, \varepsilon \cup \rho \in \dot{\mathbb{P}} \text { ", }
$$

which completes the construction.

Let $\xi \in J_{N-1}^{0}$. Then there exists $u \in S$ such that

$$
u \Vdash_{S} " \dot{\tau}_{i_{N-1}^{0}}^{\xi} \in \dot{K}_{N-1}^{0} " .
$$

Then by our construction, $s_{i_{N-1}^{0}}<_{S} t_{i_{N-1}^{\xi}}^{\xi} \leq_{S} u$ and for all $\mu<N-1$,

$$
\psi_{t_{i_{N-1}^{\xi}}^{\xi}, t_{i_{\mu}^{0}}^{\xi}}(u) \Vdash_{S} “ \dot{\tau}_{i_{\mu}^{0}}^{\xi} \in \dot{K}_{\mu}^{0} " .
$$

Since $\dot{K}_{N-1}^{1}$ is forced to be uncountable by $u$, there are $v \in S$ and $\eta \in \omega_{1}$ such that $u<_{S} v, \alpha_{\xi}<\operatorname{lv}(v), \xi<\eta, \operatorname{lv}(u)<\alpha_{\eta}^{\prime}$, and

$$
v \Vdash_{S} " \dot{\tau}_{i_{N-1}^{1}}^{\eta} \in \dot{K}_{N-1}^{1} " .
$$

(Then we notice that $\eta \in J_{N-1}^{1}$.) By our construction, we note that $t_{i_{N-1}^{1}}^{\eta} \leq_{S} v$ and for all $\mu<N-1$,

$$
\psi_{t_{i_{N-1}^{1}}^{\eta}, t_{i_{\mu}^{1}}^{\eta}}(u) \Vdash_{S} “ \dot{\tau}_{i_{\mu}^{1}}^{\eta} \in \dot{K}_{\mu}^{1} " .
$$


Since

$$
t_{\xi}=t_{0}^{\xi}=t_{i_{N-1}^{0}}^{\xi} \leq_{S} u<_{S} v, \quad t_{\eta}=t_{0}^{\eta}=t_{i_{N-1}^{1}}^{\eta} \leq_{S} v
$$

and

$$
\operatorname{lv}\left(t_{\xi}\right)=\alpha_{\xi} \leq \operatorname{lv}(u)<\alpha_{\eta}^{\prime}<\alpha_{\eta}=\operatorname{lv}\left(t_{\eta}\right) \leq \operatorname{lv}(v),
$$

it holds that

$$
t_{\xi} \leq_{S} u<_{S} t_{\eta} \leq_{S} v .
$$

For each $w \in \operatorname{dom}\left(f_{\eta}\right) \backslash S_{\leq \delta}$, there exists $\mu_{w} \in N$ such that $w \leq_{S} t_{i_{\mu_{w}}^{1}}^{\eta}$. Then since $\delta<\alpha_{\xi} \leq \operatorname{lv}(u)<\alpha_{\eta}^{\prime} \leq \operatorname{lv}(w)$, by our $\Delta$-system refinement, it holds that

$$
\begin{aligned}
& t_{i_{\mu_{w}}^{0}}^{\xi}=s_{i_{\mu_{w}}^{0}} \cup\left(t_{i_{\mu_{w}}^{0}}^{\xi}\left\lceil\left[\delta, \alpha_{\xi}\right)\right)=s_{i_{\mu_{w}}^{0}} \cup\left(t_{0}^{\xi}\left\lceil\left[\delta, \alpha_{\xi}\right)\right)=s_{i_{\mu_{w}}^{0}} \cup\left(t_{\xi}\left\lceil\left\lceil\delta, \alpha_{\xi}\right)\right)\right.\right.\right. \\
& \leq_{S} s_{i_{\mu_{w}}^{0}} \cup\left(t_{\eta}\left\lceil\left\lceil\delta, \alpha_{\xi}\right)\right)=s_{i_{\mu_{w}}^{0}} \cup\left(t_{0}^{\eta}\left\lceil\left[\delta, \alpha_{\xi}\right)\right)=s_{i_{\mu_{w}}^{0}} \cup\left(t_{i_{\mu_{w}}^{0}}^{\eta}\left\lceil\left[\delta, \alpha_{\xi}\right)\right)\right.\right.\right. \\
& =s_{i_{\mu_{w}}^{1}} \cup\left(t_{i_{\mu_{w}}^{1}}^{\eta}\left\lceil\left[\delta, \alpha_{\xi}\right)\right) \leq_{S} t_{i_{\mu_{w}}^{1}}^{\eta} ;\right.
\end{aligned}
$$

hence

$$
t_{i_{\mu_{w}}^{0}}^{\xi}<_{S} w<_{S} t_{i_{\mu_{w}}^{1}}^{\eta} \leq_{S} \psi_{t_{i_{N-1}^{1}}^{\eta}, t_{\mu_{w}^{1}}^{\eta}}(v)
$$

and

$$
\begin{aligned}
\psi_{i_{i_{N-1}^{0}}^{\xi}, t_{i_{\mu_{w}}^{0}}^{\xi}}(u)=t_{i_{\mu_{w}}^{0}}^{\xi} \cup\left(u \uparrow\left[\operatorname{lv}\left(\alpha_{\xi}\right), \operatorname{lv}(u)\right)\right)= & t_{i_{\mu_{w}}^{0}}^{\xi} \cup\left(t_{\eta} \uparrow\left[\operatorname{lv}\left(\alpha_{\xi}\right), \operatorname{lv}(u)\right)\right) \\
& =t_{i_{\mu_{w}}^{1}}^{\eta}\left\lceil\operatorname{lv}(u) \leq_{S} \psi_{t_{i_{N-1}^{1}}^{\eta}, t_{i_{\mu_{w}}^{1}}^{\eta}}(v)\right.
\end{aligned}
$$

It follows that

$$
\psi_{t_{i_{N-1}^{1}}^{\eta}, t_{i_{\mu_{w}}}^{\eta}}(v) \Vdash_{S} " \dot{\tau}_{i_{\mu_{w}}^{0}}^{\xi} \in \dot{K}_{\mu_{w}}^{0} \text { and } \dot{\tau}_{i_{\mu_{w}}^{1}}^{\eta} \in \dot{K}_{\mu_{w}}^{1} "
$$

so by the construction of $S$-names $\dot{K}_{\mu_{w}}^{0}$ and $\dot{K}_{\mu_{w}}^{1}$ and the property of the club $C$,

$$
w \Vdash_{S} " \bigcup\left\{\dot{\sigma}_{\gamma} ; \exists t^{\prime} \in \operatorname{dom}\left(f_{\xi}\right)\left(t^{\prime} \leq_{S} w \& \gamma \in f_{\xi}\left(t^{\prime}\right)\right)\right\} \in \dot{\mathbb{P}} " .
$$

Therefore $f_{\xi} \cup f_{\eta}$ is a condition of $\mathbb{Q}_{S}$; moreover, $\left\langle f_{\xi} \cup f_{\eta}, t_{\eta}\right\rangle$ is a common extension of $\left\langle f_{\xi}, t_{\xi}\right\rangle$ and $\left\langle f_{\eta}, t_{\eta}\right\rangle$.

\section{ACKNOWLEDGEMENTS}

This work was almost completed when the author stayed in Vienna to attend the ESI workshop on large cardinals and descriptive set theory, June 14 to 27, 2009. I would like to thank the organizers of this workshop and Jörg Brendle and Stevo Todorčević for discussions on this work during the workshop.

I would also like to thank Katsuya Eda for his encouragement on the work related to section 3, Franklin D. Tall for letting me know the result in [2], and the referee for a careful reading and many kind comments. 


\section{REFERENCES}

[1] K. Devlin and S. Shelah. A weak version of $\diamond$ which follows from $2^{\aleph_{0}}<2^{\aleph_{1}}$. Israel J. Math. 29 (1978), no. 2-3, 239-247. MR0469756 (57:9537)

[2] P. Eklof and S. Shelah. A combinatorial principle equivalent to the existence of non-free Whitehead groups. Abelian Group Theory and Related Topics (Oberwolfach, 1993), 79-98, Contemp. Math., 171, Amer. Math. Soc., Providence, RI, 1994. MR1293134 (95g:20056)

[3] I. Farah. $O C A$ and towers in $\mathcal{P}(N) /$ fin. Comment. Math. Univ. Carolin. 37 (1996), no. 4, 861-866. MR1440716 (98f:03043)

[4] B. König. Trees, Games and Reflections, Ph.D. thesis (2002) at the Ludwig-MaximiliansUniversität München.

[5] P. Larson and F. Tall. Locally compact perfectly normal spaces may all be paracompact, preprint.

[6] P. Larson and S. Todorčević. Chain conditions in maximal models. Fund. Math. 168 (2001), no. 1, 77-104. MR1835483 (2002e:03067)

[7] P. Larson and S. Todorčević. Katětov's problem. Trans. Amer. Math. Soc. 354 (2002), no. 5, 1783-1791. MR:1881016 (2003b:54033)

[8] T. Miyamoto. $\omega_{1}$-Souslin trees under countable support iterations. Fund. Math. 142 (1993), no. 3, 257-261. MR 1220552 (94f:03060)

[9] S. Todorčević. Partition Problems in Topology. Contemp. Math., 84, Amer. Math. Soc., Providence, Rhode Island, 1989. MR.980949 (90d:04001)

[10] S. Todorčević. Chain conditions in topology. II, in preparation.

[11] S. Todorčević and B. Veličković. Martin's axiom and partitions. Compositio Math. 63 (1987), no. 3, 391-408. MR909386 (89a:03094)

[12] T. Yorioka. Some weak fragments of Martin's axiom related to the rectangle refining property. Arch. Math. Logic 47 (2008), no. 1, 79-90. MR2410821 (2009e:03097)

[13] T. Yorioka. The inequality $\mathfrak{b}>\aleph_{1}$ can be considered as an analogue of Suslin's Hypothesis. Axiomatic Set Theory and Set-Theoretic Topology (Kyoto 2007), Sūrikaisekikenkyūsho Kōkyūroku No. 1595 (2008), 84-88.

[14] T. Yorioka. Rudin's Dowker space in the extension with a Suslin tree. Fund. Math. 201 (2008), no. 1, 53-89. MR2439023 (2009h:03067)

[15] T. Yorioka. A non-implication between fragments of Martin's Axiom related to a property which comes from Aronszajn trees, Ann. Pure Appl. Logic 161 (2010), no. 4, 469-487.

Department of Mathematics, Shizuoka University, Ohya 836, Shizuoka, 422-8529, JAPAN

E-mail address: styorio@ipc.shizuoka.ac.jp 